\title{
Pathological blood coagulation and the mycotoxic oxidative stress test (MOST)
}

Volume 2 Issue 6 - 2016

\section{Introduction and historical perspective}

More than 150years ago, British physician T. W. Jones asked the question, "Why does the blood circulating in the vessels not coagulate?". ' though a general answer to this question is now obvious, the biochemical mechanisms involved in how the blood coagulates (clots) are complex and varied, and all the intricacies have not yet been explained. A. Trousseau recognized that the blood of cancer patients is in a hyper-coagulable state in the process of coagulation, even while confined in the blood vessels. ${ }^{2}$ The name given to this discovery is still in use today, as "Trousseau's Syndrome". ${ }^{2}$ Early in his career, Rudolph Virchow, the Father of Pathology, was interested in thrombosis and embolism. He speculated that intravascular blood could be altered so it would clot as a result of a stimulus too weak to clot normal blood. ${ }^{3}$ In 1856 Virchow delivered a lecture setting forth this concept.

Although the concept of partial clotting within vessels reaches back to the beginnings of modern medicine, much of the discovery of its biochemical mechanisms-the activation of clotting factors-has been left to chance. The admission of a patient to the hospital with an unceplained bleeding disorder challenged researchers to discover the cause of hemorrhaging. Analysis of blood from normal persons helped in the study of the patient with the blood disorder. A new clotting factor was hereby discovered which was missing from the patient's blood. For this reason, several clotting factors have been named after the individuals in which they were missing: e.g., Christmas factor (factor IX), ${ }^{4}$ Hageman factor (factor XII). ${ }^{4}$

In this article, the causes of pathological (intravascular) clotting will be described, as will various methods of detecting this condition, especially a blood test I call the Mycotoxin Oxidative Stress Test (MOST).

\section{The mechanics of blood coagulation}

Blood clotting is a highly detailed chemical-mechanism involving many distinct components. The problem for the hematologist has been to understand it at the biochemical level. Undoubtedly, efforts to fully understand blood clotting will continue for many more years.

Recalling Antione Bechamp's ${ }^{5}$ and Gunther Enderlein's ${ }^{6}$ research into the sub cellular living elements and combining this with what is known of colloidal flocculation, ${ }^{7}$ it is suggested that the clotting of blood begins with the end-linking (polymerizing) of the fundamental protein unit called by Be champ the microzyma. ${ }^{5}$ A chain of these living units constitutes fibrinogen, which is still dispersed 9microhetergenous 0 in the blood, and it may or may not be further processed. If processing continues, it will be either by continued end-linking or by cross-linking. End-linked fibrinogen is referred to here as fibrin monomer, which I have suggested is a repair protein also dispersed in the blood. Due to a number of blood clotting factors, the process may continue until the excess fibrin monomer and/or until fibrin becomes excessively end-linked.

\author{
Robert OYoung \\ Universal Medical Imaging Group, USA
}

Correspondence: Robert O Young, pH Miracle Inc., 16390 Dia del Sol,Valley Center, California, 92082, USA, Tel 760 75I 832I, Email phmiracleliving@aol.com

Received: December 07, 2015 | Published: September 20, 2016
Cross-linking the polymerized strands to form a three-dimensional network results in what is called the hard clot (fibrin - the major protein of clotting blood). Factor XIII, which instigates the forming of these blood networks. Is always present but latent in the blood, and must be activated before the formation can occur. Persons who are producing fibrin monomer or excessively linked fibrinogen are said to be in a hyper-coagulable state, while those having diminished ability to form clots are in a hypo-coagulated state. It is the activation of the colloidal clotting factors which is so complex. Blood clotting may occur through many pathways and be initiated by many different stimuli. Regardless of initiation factors, the process is a sequence of events in which the activation of one factor triggers another, until, after a series of discrete steps, fibrin is formed.

When blood is clotted prematurely, and the factors involved are consumed (incorporated into) the body recognizes a deficiency of clotting agents and generates more. Thus, people with a tendency to clot excessively will alternate between a hyper coagulable state and a hypo-coagulatable state. When in the hypo coagulated state, such people hemorrhage until the deficient clotting factors are replaced. ${ }^{4}$ When only fibrin monomer or excessively linked fibrinogen is formed (no cross-linking), it is quite subtle and may go undetected. It may be detected by a change in blood viscosity (sedimentation rate), by the Mycotoxic Oxidative Stress Test (described later), or by other more subtle means. If strands of fibrinogen are cross-linked, however, a suggicient amount of insoluble precipitate of fires may result, and these can be detected microscopically using a phase contrast and dark-field microscopy in prepared slides of fresh tissue or blood. An excessive formation of fibrin leads to an impairment in circulation, and eventual organ failure usually results. ${ }^{8}$

With this background, we are in a position to consider a standard medical term: disseminated intravascular coagultion (DIC). ${ }^{7}$ This term encompasses the hyper coagulable state, I refer to as pathological blood coagulation which consists of both insoluble and excess dispersed polymers of colloidal proteins. 


\section{Key ingredients of pathological blood coagulation}

Before discussing DIC in more detail, it is necessary to introduce its fur important ingredients according to this view - mycotoxins, endotoxins, exotoxins, and tissue factor. Any of these elements, or any combination of them, can play a major role in initiating unwanted DIC. ${ }^{7}$ However, mycotoxins or the acids from yeast have been found to be the underlying element which instigates and intensifies the participation of the other three. ${ }^{6}$ Each will now be described in turn and brought into the clotting picture (Monograph 1).

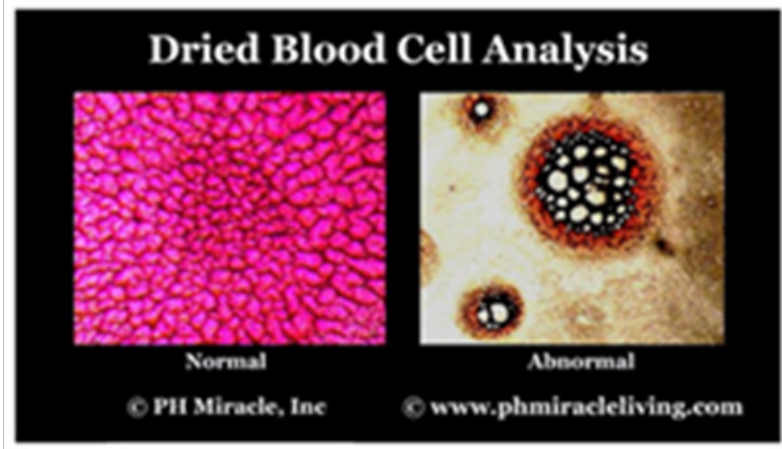

Monograph I Left, shows normal hyper-coagulated blood in a healthy blood clot sample and right, hypo coagulated blood in an unhealthy blood clot sample.

\section{Mycotoxins and metabolism by fermentation}

As discussed in the main text of my published book, Sick and Tired book. ${ }^{9}$ Acidification of blood and body tissues and organs and the accompanying lack of oxygen lead to pathological metabolic fermentation, which is carried out primarily by yeast and mould. Such pathological microorganisms, or their precursors, are inherent to the human body and to all higher organisms. Their precursors according to Bechamp, the microzymas, carry on a nominal and homeostatic fermentation themselves. Under healthy conditions. ${ }^{5}$ The primary function of yeast and mould is to decompose the body upon the death of the animal or human organism. Their premature overgrowth indicates a biochemical environment akin to death. During pathological metabolic fermentation, high concentrations of several acidic substances called mycotoxins are created. They are highly damaging, always acidic, metabolic products. If not immediately buffered by specific antioxidants, such as hydrogen peroxide and the hydroxyl free-radical, mycotoxins can seriously disrupt the physiology by disrupting normal metabolism and by penetrating blood and body cells and poisoning them. As will be seen, they interact with many of the mechanisms for DIC in various pathological symptomologies.

In my published article called The Finger on the Magic of Life: Antoine Bechamp, 19th Century Genius (1816-1908), I discuss pleomorphism in some detail. ${ }^{9}$ Understanding this phenomenon - the rapid evolution of microorganisms across traditional taxonomic lines is helpful in getting a complete picture of DIC. Briefly stated, colloidal living microzymas evolve intracellularly into more complex forms (microorganisms), beginning with a healthy primitive stage comprising of repair proteins. As the disease condition worsens, morbid intermediate forms (filterable bacteria or viruses, cell-wall deficient forms and full bacteria) develop from repair proteins, or directly from microzymas. A third macro stage comprises the commonly recognized culminate microorganisms which are yeast, fungus to mould. In terms of pleomorphism, all of these microorganisms represent a single family of variously functioning forms. ${ }^{5}$ The culminate forms produce the lion's share of acids, which are mycotoxins and the primary focus of my research. ${ }^{5,69}$ For convenience, bacteria, yeast, fungus and mold that produce acidic metabolic wastes and protein cellular fragments called exotoins, endotoxins and mycotoxins will here be referred to collectively ash EMPO, or exotoxic, mycotoxic-producing microorganisms.

What follow is a shortened description or the description and origin of several exotoxins and mycotoxins, referred to collectively microzymian acidic toxins of MAT, which are involved in the processes leading to DIC. The bio-effects or the pathology of cellular fermentation, of these toxic metabolites are known as mycotic illness, mycotoxicosis, or mycotoxic stress as seen in the MOST and described and published by Dr. Bolin in the 1940's. ${ }^{10}$

One such metabolic product is acetyl aldehyde, which is formed by cellular breakdown of food, especially carbohydrate and the birth of EMPO. Acetyl aldehyde can also break down into a secondary substance known as ethyl alcohol. Although acetyl aldehyde presents an immediate hazard to health and well-being, nature has provided a means of buffering of neutralizing this acidic by-product of cellular digestion and fermentation almost as soon as it is created. ${ }^{11}$ The controls of acetyl aldehyde (and ethyl alcohol) are the sulfur amino acids, cysteine, taurine, methionine and the peptide glutathione which is found in red blood cells and almost all cells utilizing oxygen. ${ }^{12}$ In an attempt to buffer or neutralize MAT, the body will also bind or chelate both fats and minerals to them. ${ }^{12}$

Another member of the MAT family is uric acid, which is formed by the digestion of protein and the creation of EMPO. ${ }^{13}$ Uric acid can also break down into secondary substance, one of which is alloxan. ${ }^{14}$ This has been shown to damage the insulin-producing pancreatic beta cells leading to diabetes Monograph $1 \& 2$.

A shortage of alkalizing nutrients or an excess of MAT initiates an immune response in which a special class of free radicals which I call microzymian oxidative buffering species (MOBS) are released. ${ }^{15}$ These oxygen metabolites carry unpaired electrons and are intended to disrupt bacteria, yeast, fungus and mould, and buffer exotoxins, endotoxins, and mycotoxins. Current medical savants believe that they can disrupt just about anything they contact, including healthy cells and tissue: this is not accurate. The fact is that MOBS carriers a negative surface-charge and repel healthy cells, which also have a negative surface-charge. ${ }^{16}$ It is the positively surface-charged bacteria, yeast/fungus, mould, exotoxins, endotoxins, and mycotoxins that MOBS bind too. ${ }^{17}$ This aspect gives some insight into autoimmune phenomena, which are not, as is often maintained, the result of an overburdened immune system. They result either as a side-effect of the immune system's attempt to remove foreign or toxic elements, or as a direct attempt by the immune system to remove cells or tissue rendered useless or disturbing to the body by MAT.

In every degenerative symptomatology I have studied, I have found excessive MAT and MOBS (Tables 1-3). Some of these degenerative symptoms and their underlying disease conditions, including cancer are described in my recently published paper on a deficiency on alkaline nutrition and cancer. ${ }^{18}$ But the fact that mycotoxins cause harm to humans and other animals is purely a secondary effect, since, as noted, the primary function of the microorganism is not to cause illness. We know from the fossil record that pleomorphic microforms existed long before animals. ${ }^{19}$ In fact, humans and animals developed in terms of microorganisms. ${ }^{20}$ The reverse, however, is not true. Since microorganisms appeared first in the developmental sequence, they are not physiologically aware of humans and animals. There is much 
evidence that human and animal physiologies are highly aware of, and respond to MAT - these acidic compounds signaling the presence of bacteria, yeast, fungi and/or mould or EMPO. ${ }^{2}$

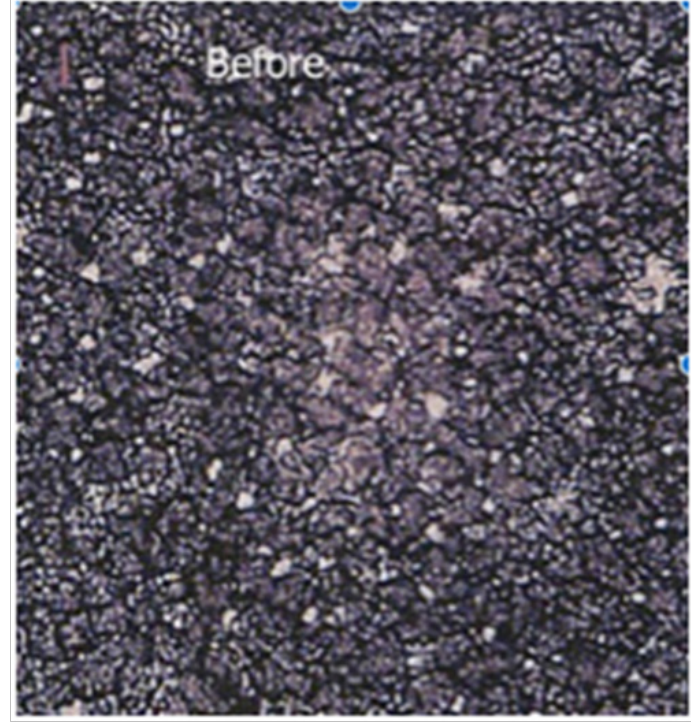

Monograph 2 An Anxiety Profile showing a 'snowstorm' of 2 to 10 micron protein polymerizations starting from the center of the clot and moving out towards the edge.

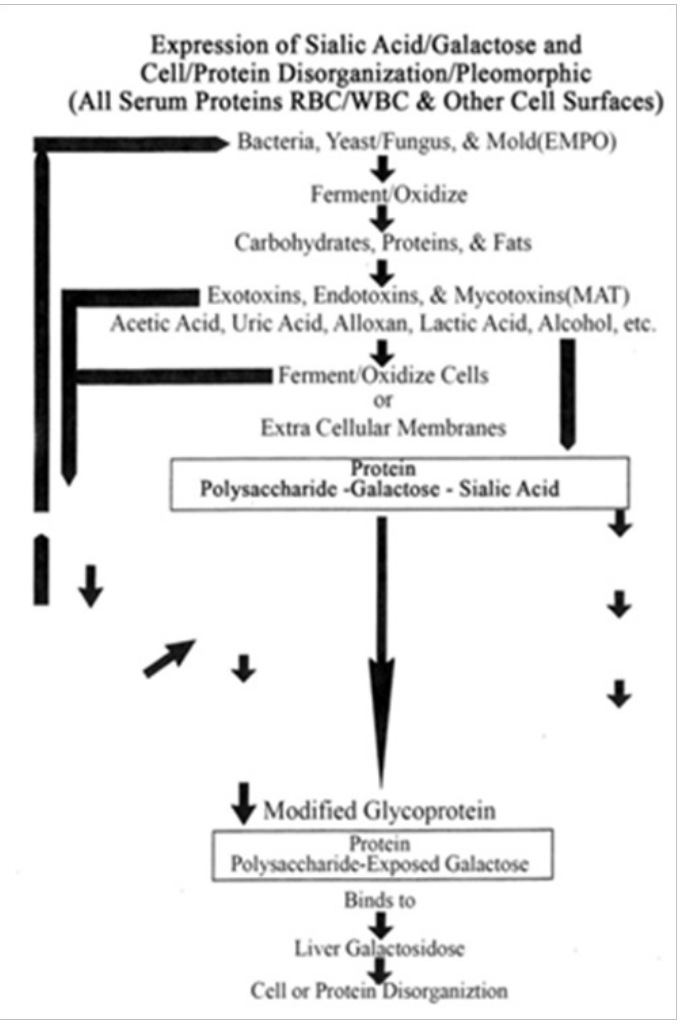

\section{Endotoxins}

Also involved in the process leading to DIC are endotoxins, substances endogenous to symptogenic (i.e., "pathogenic" in orthodox terms) bacteria. Endotoxins are a family of related substances having certain common characteristics, but differing from one bacterial form (or strain) to another. Endotoxins are lipopolysaccharides (LPS). LPS form a widely diversified group because of ${ }^{1}$ the number of long- chain fatty acids composing lipids; ${ }^{2}$ the number of individual sugars as well as their modes of linkage to one another; ${ }^{3}$ the branching of sugar chains; and ${ }^{4}$ the number of possible arrangements of these units. Endotoxins also contain proteins, further compounding the structural diversity. ${ }^{22}$

\section{Expression of Sialic Acid from Pancreatic Beta-Cells \& Type I/Type II}

\author{
Normal \\ Pancreatic Beta-Cells with no \\ or minimal surface Sialic Acid \\ $\checkmark$ \\ Regulation of \\ Insulin Production
}

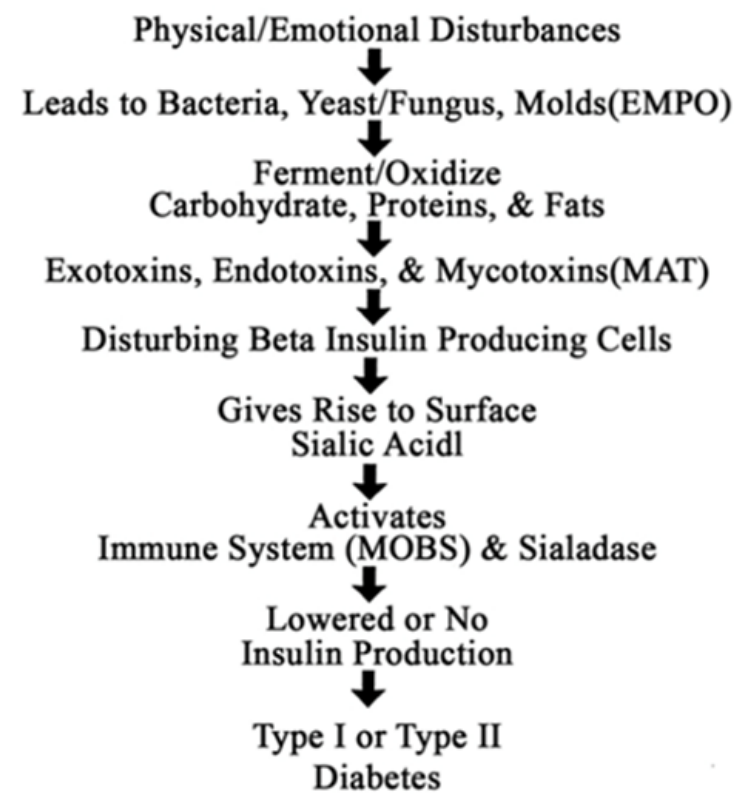

The Beta or Insulin-producing cells of the Islets of Langerhans express Sialic Acid on their surfaces.

I have shown that when Beta cells are disturbed and begin to disorganize that sialic acid is released and insulin production stops.

One theory on endotoxin states that its purpose is to act as a semipermeable membrane for the bacterium, limiting and regulating substances entering the organism. ${ }^{22}$ Endotoxin resides solely on or near the interior surface of the cell membrane and is shed into the surrounding medium only upon the death of the bacterium. Thus, as these microforms die off, or are lysed by bodily activity, endotoxin is released. (This fact may well be an explanation for the Herxheimer reaction, in which a patient becomes worse following the administration of toxic drugs or other forms of treatment that drastically alter the associated organism. ${ }^{22}$ Another endotoxin theory states that LPS are a constituent of the membrane, and as the organism grows, endotoxin fragments are repeatedly sloughed off into the medium. This phenomenon has been observed in the digestive tract. ${ }^{22}$ Since bacterial translocation into the blood is not only possible but common where epithelial hyperpermeability exists, one can assume that the process will continue there. Both theories may be correct if we think of the first one as true of "adult" forms, and the second as true of newly developed and expanding ones. 
Basic to the structure of an endotoxin is the lipid common to all forms, designated lipid A, to which is attached a "core" polysaccharide, identical for large groups of bacteria. To the core polysaccharide is attached the O-antigen, consisting of various lengths of polysaccharide chains which are chemically unique for each type of organism and LPS. These chains provide endotoxin specificity. ${ }^{23}$ Experiments conducted over many years indicate that most, if not all, of the toxic effects of an endotoxin may be attributed to the lipid portion, and it is sometimes used per se in experiments rather than the entire molecule. ${ }^{24}$ An important additional feature of lipid A is its phosphate content. Each phosphate group carries a negative charge, and since lipid A is a rather large molecule, it provides, essentially, a negatively charged surface. The importance of this will be seen shortly.

\section{Heart Attacks, Strokes, Congestive Heart Failure High Blood Pressure \& Atherosclerosis Hypothesis}

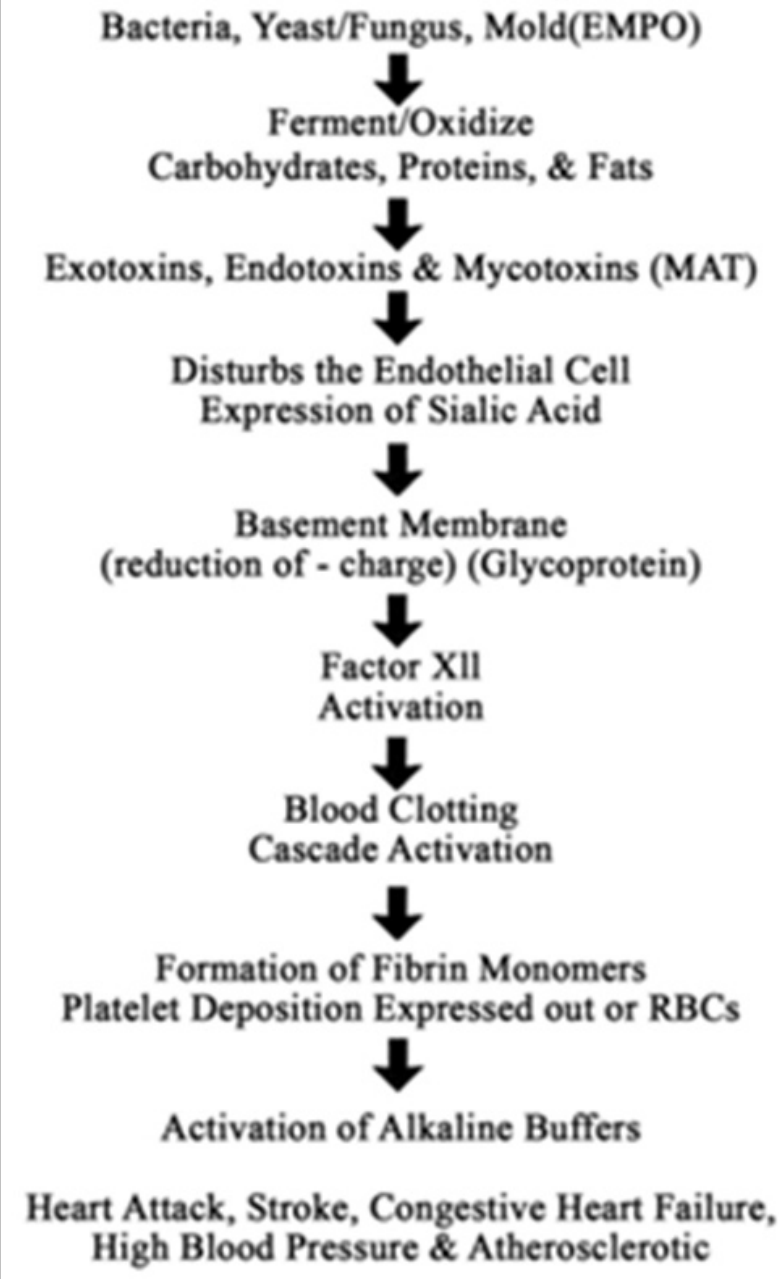

\section{Exotoxins}

These are the metabolic excretions of bacteria. While endotoxin's ongoing effect is, in a manner of speaking, in the background, exotoxins, like mycotoxins, present a double-edged sword. Not only do they initiate DIC, but they produce, or influence the body to produce, the various and numerous infectious symptomatologies, such as typhoid fever, diphtheria, etc. (See "Vaccination Reconsidered" in Section 4 of the Appendix of Sick and Tired for details on the action of diphtheria toxin). ${ }^{6}$ By comparison, mycotoxins not only initiate DIC, but there is much evidence to suggest that they produce, or influence the body to produce, degenerative symptomatologies, such as arthritis, diabetes, etc., and cancer and AIDS as well.

Table I Expression of Sialic Acid/Galactose MAT from Cell and Protein Degeneration (From All Serum Proteins, RBC/WBC and Other Cell Surfaces

Carbohydrate, Proteins, and Fats From Diet, Body Cells or Reserves

As cells breakdown or ferment they give birth to bacteria, yeast, fungus and mold [EMPO] and their associated metabolic acidic waste [MAT]

Exotoxins, Endotoxins, and Mycotoxins MAT

Acetyl Aldehyde, Ethyl Alcohol, Uric Acid, Alloxan, Lactic Acid are examples of MAT

MAT Ferments Other Body Cells and their Extracellular Membranes and Proteins

MAT Modifies Glycoprotein

Binds to liver Galactosidase

Creating an Increase in Cell and Protein Fermentation and Degeneration and Increased Amounts of Exotoxins, Endotoxins and Mycotoxins MAT

Table 2 Expression of Sialic Acid MAT From the Fermentation of Degeneration of Insulin Producing Pancreatic Beta-Cells in Type I,Type II and Type III Diabetes

Pancreatic Insulin producing Beta-Cells with no or

minimal Surface Sialic Acid MATA Physical and/or Emotional Disturbance Occurs from Lifestyle and/or Diet

Normal regulation of Insulin Production

A Physical and/or Emotional Disturbance Occurs from Lifestyle and/or Dietary choicesdd

Leads to cellular fermentation and degeneration and the birth of EMPO This lead to increased abnormal amounts of MAT that the immune system, the alkaline buffering system and the elimination organs has to deal with Fermenting and degenerating Insulin Producing Beta Cells

Giving Rise to Surface Cell Sialic Acid [MAT\}

Increased Amounts of Sialic Acid Activates the Immune Response MOBS and Sialidase $A B$

Leads to Lowered or No Insulin Production

Symptoms of Type I, Type II or Type III Expressed

The insulin producing beta cells of the Islets of Langerhans express silica acid on their surface as a break

down metabolite. I have suggested that when insulin producing beta cells are physically disturbed by MAT

they begin to disorganize and express sialic acid on the surface of the cell.

This indicates the death of the cell

and insulin production will stop.

\section{Tissue factor}

Crucial to the understanding of DIC is recognition of the role of tissue factor (TF), formerly known as thromboplastin. This transmembrane lipoprotein exists on the surface of platelets, vascular endothelial cells, leukocytes, monocytes, and most cells producing EMPO ${ }^{24}$ It plays a major role in several biochemical mechanisms leading to DIC.

TF is the primary cell-bound initiator of the blood coagulation cascade. Its gene is activated in wound healing and other conditions. By itself it is capable of initiating clotting, but also becomes active when complexed with factor VII or activated factor VII (Vila). ${ }^{25} \mathrm{TF}$ has been described as the receptor for factor VII because of the close association between the two proteins and because it causes a shape change (conformational) in factor VII, allowing it to attain activity. 
Both factor Vila and the TF/VII complex activate factors IX and X, which initiate the clotting cascade and the formation of thrombin. ${ }^{4}$

Table 3 High Blood Pressure, Atherosclerosis, Heart Attacks, Strokes, and Congestive Heart Failure

A Physical and/or Emotional Disturbance Occurs from Lifestyle and/or Dietary choices

Leads to cellular fermentation and degeneration and the birth of EMPO This lead to increased abnormal amounts of MAT that activates the immune system to chelate the MAT.

Increased amounts of MAT will cause endothelial breakdown and the expression of Sialic acid.

Increased Amounts of Sialic Acid and damage to the endothelial will cause a reduction in the negative surface-charge leading to the release of Glycoproteins.

The release of Glycoproteins will cause the activation of Factor XII and the blood clotting cascade.

This cause the creation and formation of fibrin monomers and the increase of Platelet Deposition out of the red blood cells for clotting purposes

The immune system will activate and MOBS will be released as well as sodium bicarbonate, calcium, lipids and other alkaline buffers to reduce metabolic acidity

The build-up of fibrin monomers in the clotting cascade will lead to fibrin nets and clots causing an increase in blood pressure and the risk of blockages potentially causing a Stroke or Heart Attack.

\section{Development of disseminated}

\section{Intravascular coagulation (DIC)}

DIC induced by MAT and tissue factor: An infusion of toxins into the blood has a direct effect on TF gene expression in leukocytes. Contact of MAT, endotoxins (lipid A), or exotoxins with leukocytes, activates proteins that bind to DNA nucleotide sequences, thereby activating the TF gene ${ }^{4}$ (Tables 4-6).

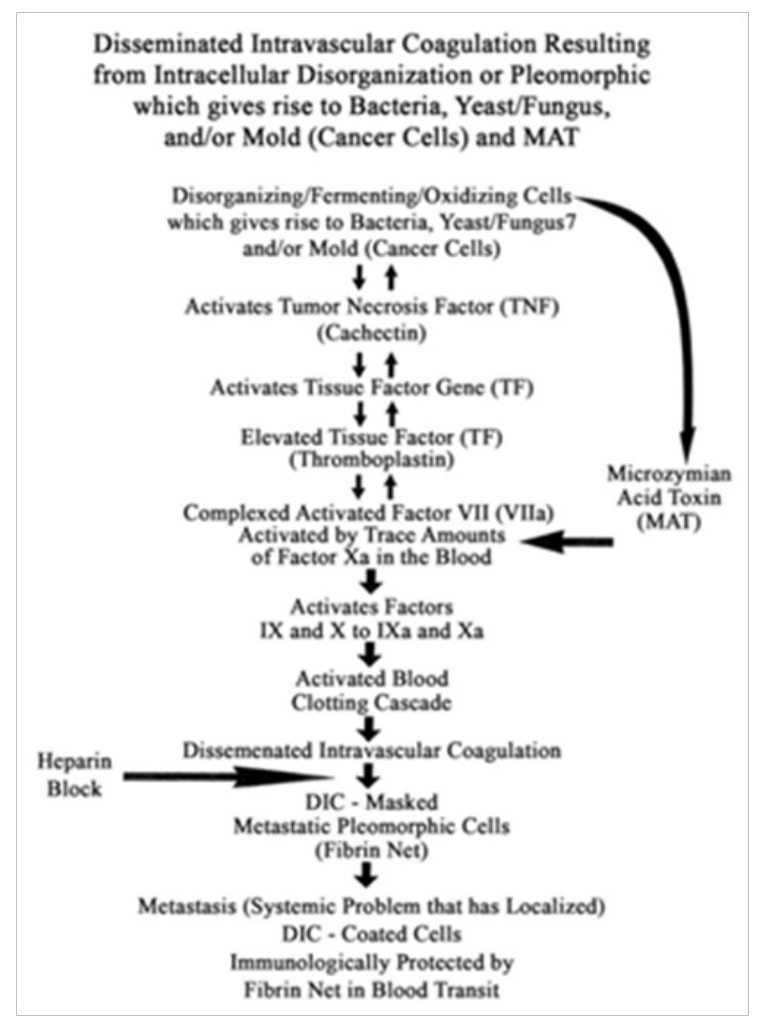

Table 4 Disseminated Intravascular Coagulation Resulting from Intracellular Disorganization or Fermentation which Gives Rise to Mat and Empo

A Physical and/or Emotional Disturbance Occurs from Lifestyle and/or Dietary choices

Leads to cellular fermentation and degeneration and the birth of EMPO

This lead to increased abnormal amounts of MAT that activates the Tumor Necrosis Factor (TNF).

Increased amounts of TNF activates the Tissue Factor Gene (TF) Increased Amounts of TF causes the release of Thromboplastin.

The release of Thromboplastin activates the release of clotting Factors VII (Vlla) and trace amounts of Factor Xa into the blood.

This activates the release of Factors $I X$ and $X$ to $I X a$ and the increase of Factor Xa.

The activation of the blood clotting cascade leads to Disseminated Intravascular coagulation and the clotting or thickening of the blood inside the blood vessels.

The DIC or hyper-coagulation will mask the fermentation of healthy cells to unhealthy cells or cancerous cells.

As the unhealthy cells or cancerous cells increase the body will go into preservation mode and begin forming fibrin nets to encapsulated these unhealthy cells to protect healthy body cells.

As body and blood cells breakdown from MAT this causes an increase of MAT and EMPO leading to systemic latent tissue acidosis and a potential metastatic cancerous condition.

Table 5 Disseminated Intravascular Coagulation Resulting in Cellular Disorganization or Fermentation and the Increase of Mat and Empo

A Physical and/or Emotional Disturbance Occurs from Lifestyle and/or Dietary choices

Leads to cellular fermentation and degeneration and the birth of EMPO

This lead to increased abnormal amounts of MAT that activates the Tumor Necrosis Factor (TNF).

Increased amounts of TNF activates the Tissue Factor Gene (TF) Increased Amounts of TF causes the release of Thromboplastin.

The release of Thromboplastin activates the release of clotting Factors VII and Factor $\mathrm{Xa}$ in the blood.

This activates the release of Factors IX and X to IXa and the increase of Factor Xa.

The activated blood clotting cascade leads to Disseminated Intravascular coagulation and the clotting or thickening of the blood inside the blood vessels.

The DIC or hyper-coagulation will mask the fermentation of healthy cells to unhealthy cells or cancerous cells.

As the unhealthy cells or cancerous cells increase the body will go into preservation mode and begin forming fibrin nets to encapsulated the unhealthy cells.

This leads to tumor formation of the unhealthy or cancerous cells.

As the body and blood cells breakdown this causes an increase of MAT and EMPO leading to an increased risk of systemic metastatic cancer.

Endothelial cells damaged in culture by exotoxins, endotoxins, or mycotoxins attract polymorphonuclear leukocytes (PMNs), which adhere to the damaged cells. Once the leukocytes are bound, they can still have their TF gene activated if it hasn't yet occurred, and they may release MOBS in response to toxins and to organisms of disease, possibly creating further disturbances. (Cellular disorganization then releases activating proteins into the blood, which is discussed in more detail later.) Research shows that exotoxic and mycotoxic stress resulting in bound PMNs can be blocked by "antioxidants". ${ }^{26}$ These might better be called anti-exotoxins or antimycotoxins. Both observation and study have led the author to conclude that cellular 
disorganization is initiated and primarily caused by fermentation pathology, not, as is the current belief, by the MOBS, or free radicals, generated to destroy toxins and microorganisms. MOBS or free radicals, because of their negative charge, are released to chelate or bind EMPO and MAT. It is suggested by current savants that free radical tissue damage is the secondary, "shotgun" effect of intense immune response to EMPO toxification and MAT-damaged cells. This could not be the case since healthy cells or their membranes carry a negative charge and would resist any electromagnetic attraction because of similar charge. The concentration and instability of MAT generated in a compromised terrain, as opposed to the fleeting existence of free radicals, especially exogenous ones, also lead to this conclusion.

Table 6 Endotheial Cell Conversion from an Antithrombotic State to a Procoagulant State Cellular Disorganizing Pathway

A Physical and/or Emotional Disturbance Occurs from Lifestyle and/or Dietary choices

Leads to cellular fermentation and degeneration and the birth of EMPO

This lead to increased abnormal amounts of MAT that activates the Tumor Necrosis Factor (TNF).

Increased amounts of TNF activates the Tissue Factor Gene (TF)

Shedded vesicles with TF coming from disorganizing fermenting body cells activates the release of clotting Factors VII (VIla) and small amounts of Factor $\mathrm{Xa}$ in the blood.

This activates the release of Factors IX and X to IXa and increased amounts of Factor Xa.

The activated blood clotting cascade leads to Disseminated Intravascular coagulation and the clotting or thickening of the blood inside the blood vessels.

The DIC or hyper-coagulation will mask the fermentation of healthy cells to unhealthy cells or cancerous cells.

As the unhealthy cells or cancerous cells increase the body will go into preservation mode and begin forming fibrin nets to encapsulate the unhealthy cells.

As body and blood cells breakdown this causes an increase of MAT and EMPO leading to systemic metastasis.

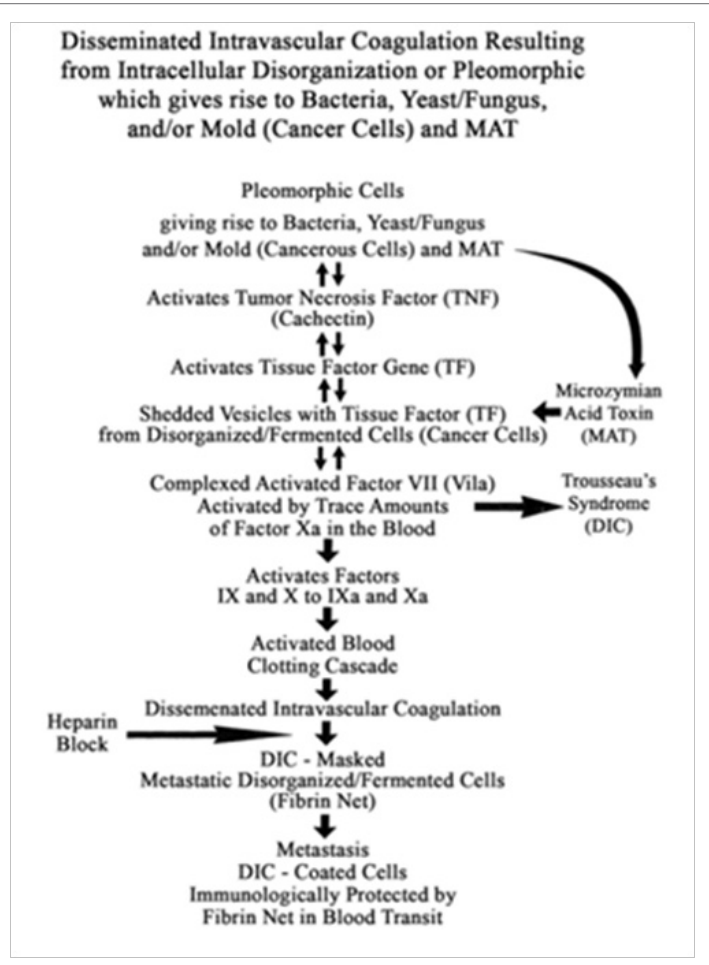

Endothelial Cell Conversion from an Antithrombotic State to a Procoagulant State Cellular Disorganizing Pathway

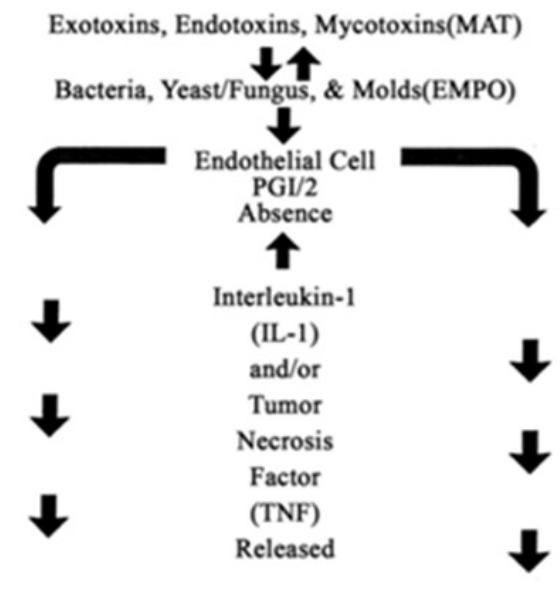

Endothelial cells grown in culture can be induced to express tissue factor. In one experiment, no procoagulant activity could be detected in the absence of toxins. However, the addition of mycotoxins from Aspergillus niger or Micrococcus neoformas (Mucor racemosus Fresen) resulted in procoagulant activity which reached a maximum in four to six hours and was dose-dependent. The same experiment was applied using E. coli and Salmonella enteritidis endotoxin with a similar result. ${ }^{27} \mathrm{~A}$ single intravenous injection of a mycotoxin from Aspergillus niger into experimental animals resulted in circulating endothelial cells within five minutes. In other experiments with the mycotoxin, detachment of endothelial cells from the basement membrane was noted ${ }^{28}$ (Table 7).

Table 7 Mechanism of Disseminated Intravascular Coagulation Generated by Mat

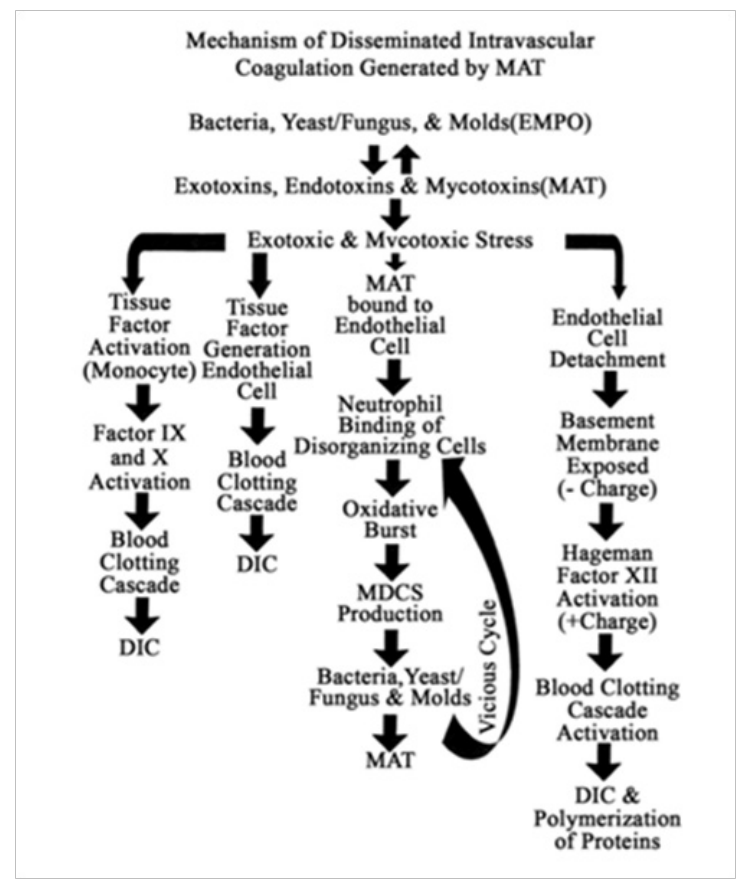


Removal of endothelial cells has dire consequences from two standpoints: First, the surface of these cells is covered with a specific prostaglandin (PGI2) known as prostacyclin. If blood contacts a surface not covered with PGI2, it will clot. For example, surfaces devoid of this prostaglandin are formed whenever a vessel is cut or punctured. An abrasion or other injury may also expose a surface on which PGI2 is lacking. The removal of endothelial cells by exotoxins or mycotoxins creates a surface devoid of PGI2, leading to blood clotting (Table 8). Secondly, disorganization of endothelial cells creates increased levels of EMPO and MAT which are attracted to an exposed surface (basement membrane) which expresses a negative charge. This also leads to clotting.

Table 8 Endothelial Cell Conversionfrom an Antithrombotic State (Norma Pathway)

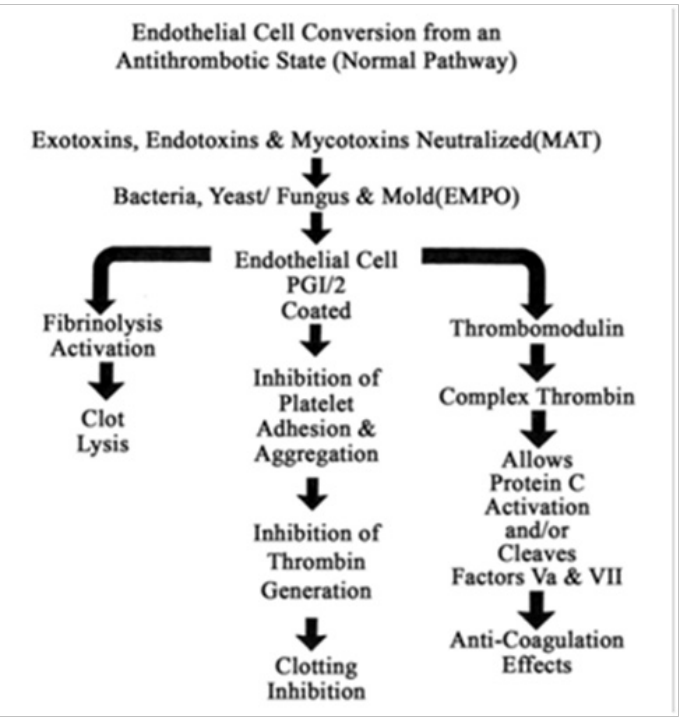

Table 9 Positive Charge of Cancerous Cells and Tumors and the Formation of Fibrin Nets and Trees in Response to Mat

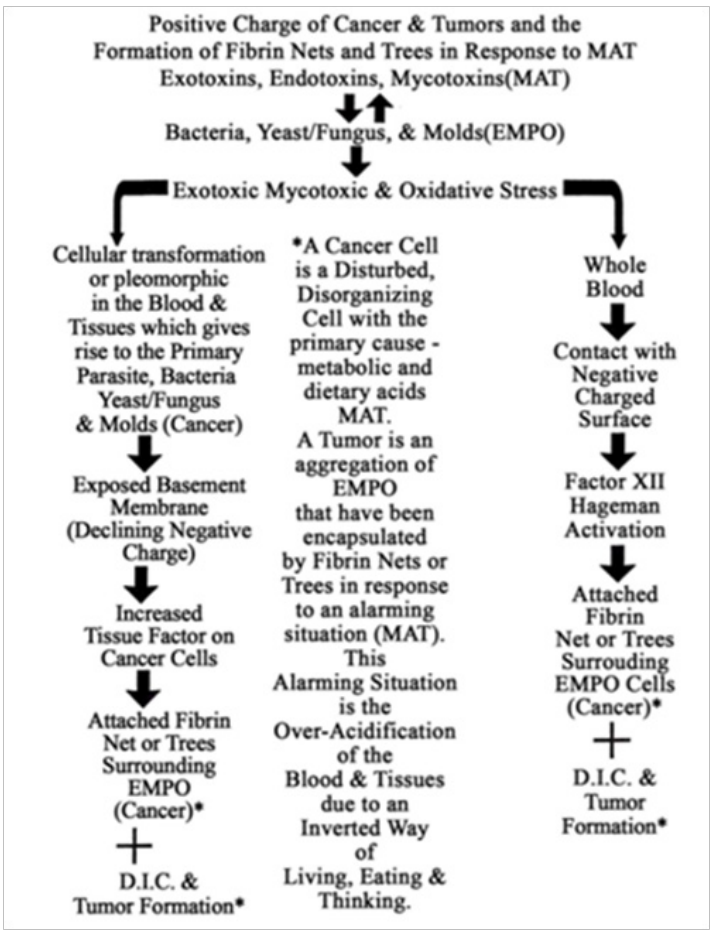

Table 10 Disseminated Intravascular Coagulation Resulting from Phagocytic Oxidative Burst

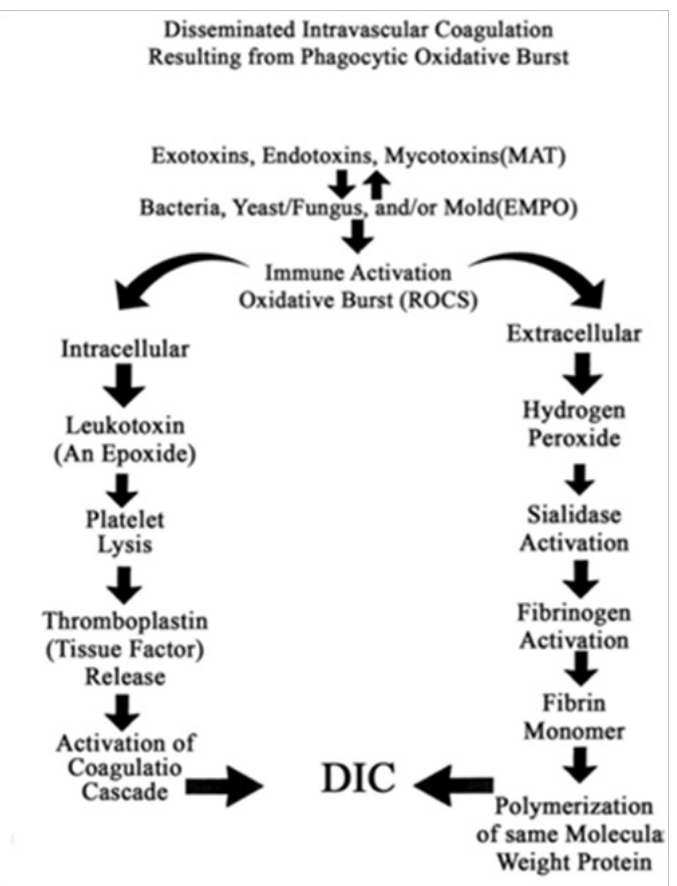

Table II Activation of Sialidase and Microzymian Oxidative Buffering Species (MOBS) by Empo and Mat

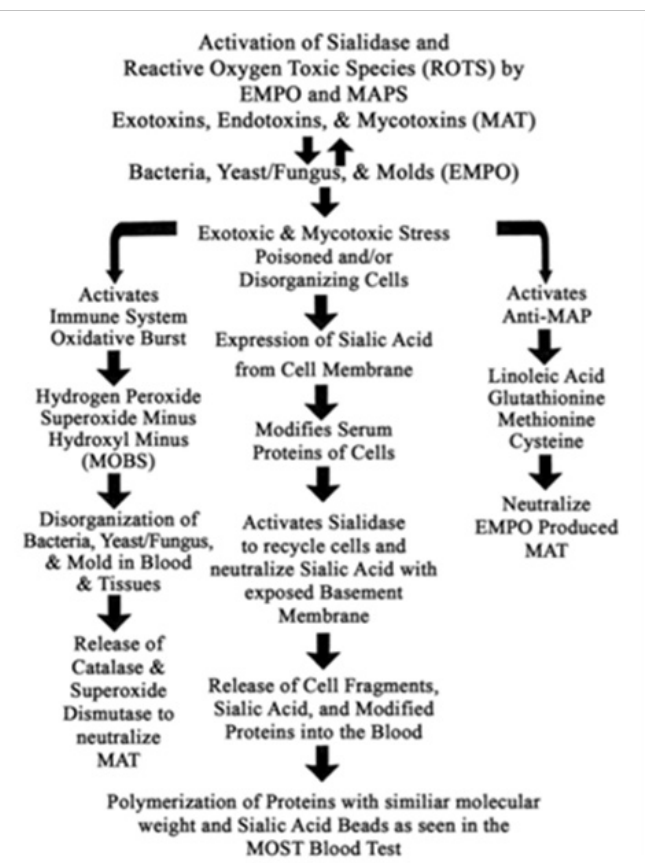

DIC induced by electrostatic attraction: It was discovered in 1964 that blood will clot simply from contacting a negatively charged surface ${ }^{29}$ Previously it was believed that the clotting process comprised a cascade of enzyme activity in which one activated the next, etc. The discovery that blood could be clotted simply by contacting a negatively charged surface ruled out the purely enzyme hypothesis. Only some of the known clotting factors have been shown to be enzymes. ${ }^{29}$ As a result of this surprising discovery, detailed research was conducted in an attempt to describe the process. In some experiments, the negatively charged surfaces of selected, finely divided, 
inorganic crystals, including aluminium oxide, barium sulphate, jeweler's rouge, quartz, and titanium oxide, were considered. ${ }^{4}$

Table I 2 Most Blood Test and Disseminated Intravascular Coagulation with Solubilized Extracellular Matrix

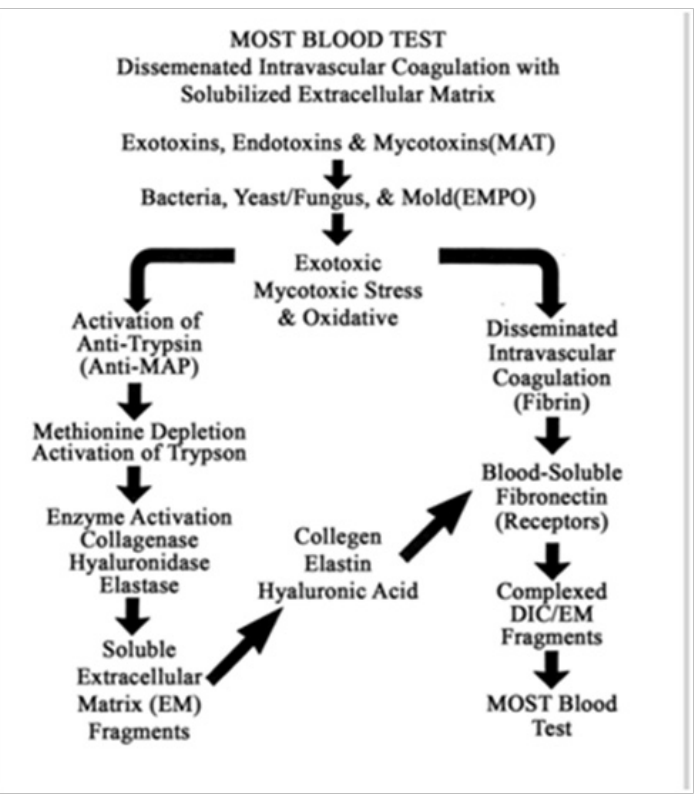

Table I 3 Typical Sources of Fermentation Insult (Mat) in Biological Systems Initiating Dic

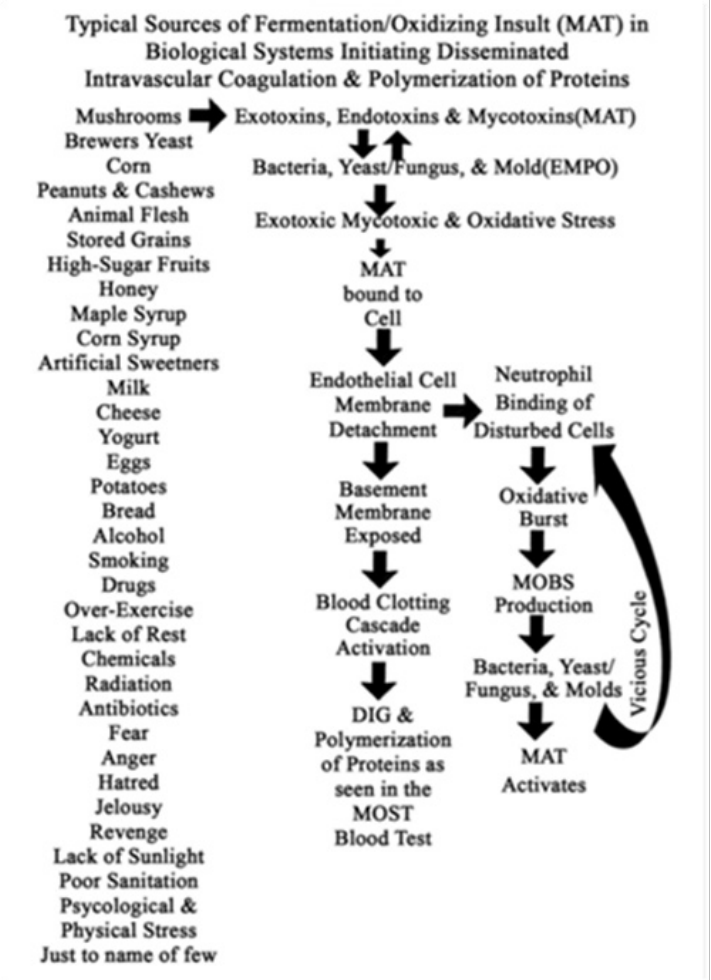

The clotting factor eventually shown to be activated when whole blood contacted negatively charged surfaces was factor XII, also known as the Hageman factor. This is a positively charged protein migrating in an electric field (electrophoresis) toward the anode. ${ }^{4}$ It is believed that factor XII is normally in the shape of a hairpin which binds to the negatively charged surface at the bend. Electrostatic attraction forces the two arms to lie flat on the surface, thereby exposing the inner faces and activating the molecule.

It was discovered that if the negatively charged particles were smaller than the clotting factor itself, activation was minimal. Or, if the concentration of clotting factor was too great, there was little or no activation. ${ }^{30}$ Both of these observations indicated that the process was one of electrostatic attraction between the negatively charged surface and the clotting factor, which is a "basic" protein, that is, positively charged. ${ }^{22,23}$

Activation of factor XII allows the activation of factor XI, which then activates factor IX. Thus, the blood clotting cascade continues to the formation of fibrin in the normal manner..$^{31}$ However, due to a series of activations begun by contact of factor XII with a negatively charged surface, trace amounts of factor Xa also show up in the blood. Factor VII is activated to Vila by factor Xa. Factor Vila then activates factors IX and X, leading to the formation of thrombin. Factor Xa, with co-factor Va, continues the clotting cascade until fibrinogen is activated, leading to fibrin formation ${ }^{4}$ (Table 5).

As discussed earlier in terms of prostacyclin, beneath endothelial cells is another surface - the basement membrane. Called the extracellular matrix, it is a thin, continuous net of specialized tissue between endothelial cells and the underlying connective tissue. It has four or more main constituents, including proteoglycans (protein/ polysac- charide). ${ }^{32}$ The removal of endothelial cells by' MAT exposes this membrane, which is negatively charged by virtue of its sulfonated polysaccharides in the proteoglycans. This brings a reduced negatively charged surface into direct contact with the blood, which activates factor XII and the clotting cascade. ${ }^{4}$ The positively charged toxic components of MAT also activate factor XII, as do disturbed disorganized cells, yeast/fungus cells, mouldy cells, and the phosphate groups in the lipid A component of endotoxin (Tables 2-5).

To summarize this section, exotoxic and mycotoxic stress resulting from overgrowth of bacteria, yeast/fungus, and then mold, has multiple actions, all leading to disseminated intravascular coagulation: MAT activation of tissue factor gene in leukocytes; subsequent activation of factors VII, IX, and X, resulting in the blood clotting cascade. MAT activation of tissue factor gene in endothelial cells, again leading to the clotting cascade.

MAT damage to endothelial cells, resulting in neutrophil attraction, with TF gene activation and generation of MOBS, which, in turn, neutralize MAT, protecting healthy endothelial cells or the basement membrane and supporting the janitorial services of the leukocytes.

Removal of negatively charged endothelial cells by positively charged exotoxins, endotoxins, and mycotoxins, creating a surface devoid of PGI2, also exposes the negatively charged basement membrane, leading to the activation of factor XII and initiation of the clotting cascade. Positively charged components of EMPO, exotoxins and mycotoxins, and several other elements, including the lipid A component of bacterial endotoxin, also activate factor XII and the clotting cascade.

Endothelial cells as antithromboticsorprocoagulants: Normal, resting (unstimulated) endothelial cells show antithrombotic activity in several ways: $:^{1}$ by the inhibition of prostacyclin (platelet adhesion and aggregation); ${ }^{2}$ the inhibition of thrombin generation; and ${ }^{3}$ the activation of the fibrinolytic system, leading to clot lysis. ${ }^{33}$ We will take a brief look at the thrombin aspect.

On the surface of endothelial cells is a protein called thrombomodulin, which acts as a receptor for thrombin. When bound to thrombomodulin, thrombin can activate protein C. Activated 
protein $\mathrm{C}$ then catalyzes the proteolytic cleavage of factors $\mathrm{Va}$ and Vila, thereby destroying their participation in blood clotting. Thus thrombin, which normally activates fibrinogen, plays an opposite role in this case and inhibits the clotting process ${ }^{33,34}$ (Table 8).

On the other side of the coin, the endothelial cell becomes a procoagulant agent when acted on by certain lymphokines, such as interleukin-1. Not only can interleukin-1 induce TF gene expression, but it also suppresses transcription of the thrombomodulin gene in endothelial cells. As in other situations, the lymphokine-activated endothelial cell expresses TF on its surface as a result of TF gene activation. This leads to the production of thrombin and the triggering of the blood clotting cascade. ${ }^{35}$ (Table 5) Many lymphokines also stimulate adhesion of leukocytes to endothelial cells damaged by MAT, resulting in recycling of the cells by MOBS, as described later.

DIC induced by intracellular fermentation by bacteria, yeast/ fungus and/or mould: Any cell which has gone from an oxidative to a fermentative state can biochemically cause macrophage production of the lymphokine tumor necrosis factor (TNF). This protein has been shown to activate the gene for $\mathrm{TF}$ in fermenting cells, which are so behaved due to morbid evolution of bacteria, yeast/fungus, and then mould ${ }^{36,37}$ In the author's view, a cell having been switched entirely to fermentation metabolism as a result of a physical or emotional disturbance of that cell, is what constitutes cancer (Tables 5 \& 9). (One might argue that this definition does not fit all "forms" of cancer, such as leukemia, for example. This is because leukemia is not cancer, but an immune response to the rise in EMPO and MAT in the body, and a relatively easy compensation to correct.)

The surface of many disorganizing or fermented cells (cancer cells) is characterized by small projections in the plasma membrane which pinch off, becoming free vesicles containing toxins as well as TF complexes with factor VII. These vesicles can aggregate and/or lodge anywhere, ultimately releasing their contents. Also, the presence of excessive amounts of TF/factor VII complexes on the surface of fermented cells allows the formation of a fibrin net around the cell and around the entire mass of cells (tumor). This seems to be an attempt by the body to encapsulate and contain the mass. However, fermented cells do escape from the primary fibrin net, perhaps due to some electromagnetic effect, and become free-floating in the circulation. They may thus lodge elsewhere and instigate the fermentation of other cells by fungal penetration or by poisoning them and provoking a morbid evolution of their inherent microzymas.

Because of the surrounding fibrin net, these mobilized fermenting cells are protected from collection by the immune system while in transit $^{38}$ (Table 4). The blockage or dissolution of fibrin net formation by an anticoagulant such as heparin allows freed, fermenting (metastasizing) cells to be dismantled by natural killer cells and other immune cells (Tables 5 \& 9).

DIC induced by MAT/EMPO and immune system response (Release of MOBS): Unsaturated fatty acids are highly susceptible to EMPO as well as MAT. Linoleic acid, a long-chain fatty acid present in white cells, has 18 carbons and 2 unsaturations. Subjected to MAT, linoleic acid binds the exotoxin, endotoxin, or mycotoxin, thereby forming an epoxide at the first unsaturation. ${ }^{39}$ Research has revealed that this compound, named leukotoxin, is highly disturbing to other cells. It causes platelet lysis, thereby releasing TF and initiating DIC ${ }^{40}$ (Table 10). The fact that MAT result in fermented fats lends further credence to the suggestion that the initial and primary degenerative damage to structures and substances in the body is caused by exotoxins and/or mycotoxins, and that damage by MOBS, or by other free radicals, is not possible.
Another mechanism leading to DIC is the release of a special glycoprotein, sialic acid, from the terminal ends of cell-membrane polysaccharides, where it is always found. Polysaccharides play a highly significant role in biochemical processes, with both enzymes and membrane receptors recognizing various groupings of specific sugars linked in highly specific ways.

Immediately preceding the release of sialic acid in the polysaccharide chain is the sugar galactose. The sialic acid/galactose arrangement is utilized as a biological indicator of cellular and molecular aging. As cells age, sialic acid is naturally expressed from the terminal ends of polysaccharides, thereby exposing galactose. A membrane-bound enzyme from the liver, galactose oxidase, recognizes galactose and eventually disorganizes it, disrupting cell function integrity and hastening demise. Aged red blood cells, which have expressed a significant amount of sialic acid, are removed from the blood by this process. (I theorize that the biological terrain may be at work in normal cell aging. That is, the rate at which sialic acid is expressed is determined by the levels of corrosive acids in the system and the body's ability to remove them, although there are no doubt intracellular factors at work as well.)

I suggest from my years of clinical research that cellular breakdown is compounded by the fermentation of the galactose by the microzyma. This is a process that begins from within and not necessarily from without. Not only does this action create more sialic acid, it creates other toxic waste products such as acetic aldehyde, alcohol, uric acid, oxalic acid, etc. The increase in cellular disturbances and fermentation of the galactose creates biochemical signals for more galactose oxidase. This leads to greater cellular disorganization and developmental morbidity, especially in the red blood cells, and a rise in the level of detrital serum proteins, which encourages clotting. From this perspective, diabetes, arthritis, atherosclerosis and other symptomatologies become more clearly "degenerative" (Tables 2-5 \& 9).

Fibrinogen is a rather elaborate protein having the structure of three beads on a string. Expressed on the end beads is sialic acid, which indicates the beginning of disorganization of the fibrinogen and a declining negative charge to the positive. Prior to the declining charge and the expression of sialic acid on the end beads, fibrinogen, which is negatively charged, will not polymerize the healthy blood due to mutual repulsion. However, fibrinogen will polymerize to damaged cells, EMPO, MAT and other positively charged areas of the body for repair purposes. Thus, as more and more sialic acid is expressed, there will be a significant reduction in the charge of the fibrinogen, acting as the primary requirement for the polymerization of fibrinogen (hypercoagulable state). The resulting polymer, fibrin monomer, is the protein chain used in the repair of cells and clotting of blood. ${ }^{41}$ End-linking will take place after the release of sialic acid (positive charge) by whatever means.

With this background, it is interesting to note that blood taken from persons suffering from anxiety is expressing sialic acid from fibrinogen, and is halfway toward clotting. Hormones released during anxiety states are easily fermented, giving more momentum to MAT and thereby resulting in this important change in fibrinogen. It leads to a clotting pattern characteristic of anxiety stress, and is readily identified in the M/OST. As can be seen in this picture, the pattern is a "snowstorm" of protein polymerizations measuring from 2 to 10 microns (Monograph 2).

As mentioned earlier, despite the attempt by the body to neutralize EMPO and MAT, an excess will initiate the release of MOBS by immune cells. A major MOBS are superoxide, designated chemically 
as $\mathrm{O}$ 2. It may exist alone or be attached to another element, such as potassium (KO'2) or sulfur (SO). Again, however, nature has provided a means of protecting healthy cells-their negative charge. ${ }^{1}$ Another protection against superoxide is the enzyme superoxide dismutase (SOD), also found in all healthy cells.

A second member of the MOBS family is hydrogen peroxide (H202). This molecule is very unstable and tends to react rapidly with other biological molecules, damaging them. The release of hydrogen peroxide in the body is a response to the overgrowth of decomposition organisms in a declining $\mathrm{pH}$ (compromised biological terrain). The control for healthy cells against hydrogen peroxide is their negative charge and the protective enzyme catalyse, one of the most efficient enzymes known.

When leukocytes and other white blood cells are stimulated by the presence of bacteria, yeast/fungus and mould, they treat these organisms as foreign particles to be eliminated. During and prior to phagocytosis, the foregoing oxidative cytotoxins, along with the hydroxyl radical ( $\left.\mathrm{OH}^{\prime}\right)$, are generated and released specifically for neutralizing microforms or harmful substances. This release is referred to as an "oxidative burst." As a result of fermentation and the production of exotoxins and mycotoxins that ferment galactose from cells, the immune system is activated. An oxidative burst is released to neutralize the morbid microforms and mycotoxicity. ${ }^{42}$ Like other biological processes faced with constantly alarming situations, the continued release of MOBS can get out of control. This may damage endothelial cells, the basement membrane, or other body elements, and this activates fibrinogen to fibrin monomer (repair protein), leading to DIC (see Table 11). Interestingly, the white blood cells capable of neutralizing MAT through MOBS production are the same ones capable of phagocytosis, the process by which foreign matter, waste products and microorganisms are collected and dumped in the liver. $^{43}$

\section{To summarize this section, morbid microforms and their acids create DIC by a number of pathways}

Leukotoxin (linoleic acid bound to mycotoxin) is highly toxic to cells. It causes platelet lysis, thereby releasing TF and initiating DIC. The expression or release of sialic acid residues from healthy cells that have been disturbed allows for the fermentation of galactose, creating exotoxins and mycotoxins, biochemically activating galactose oxidase, which further disturbs and disorganizes healthy cells. This cycle loads the blood with debris.

EMPO and MAT disturb fibrinogen, which releases sialic acid and reduces the charge, allowing it to polymerize into fibrin monomer and fibrin nets. The presence of exotoxins, endotoxins, and mycotoxins and their poisoning of cells activate the immune system. White blood cells generate MOBS (e.g., superoxide 0'2 or hydrogen peroxide H202). These substances bind to and neutralize EMPO and MAT. MOBS are repelled by healthy endothelial cells and the basement membrane because of their negative charge. Cellular disturbances and disorganization stimulate the generation of fibrin monomer for repair purposes, leading to DIC.

\section{Detection of disseminated intravascular coagulation}

\section{The sonodot analyzer}

The Sonoclot Coagulation Analyzer provides a reaction-rate record of fibrin and clot formation with platelet interaction. An axially vibrating probe is immersed to a controlled depth in a $0.4 \mathrm{ml}$ sample of blood. The viscous drag imposed upon the probe by the fluid is sensed by the transducer. The electronic circuitry quantifies the drag as a change in electrical output. The signal is transmitted to a chart recorder which provides a representation of the entire clot formation, clot contraction and clot lysis processes. The analyzer is extremely sensitive to minute changes in visco-elasticity and records fibrin formation at a very early stage. The Sonoclot has been evaluated scientifically and shown to provide an accurate measurement of the clotting process. ${ }^{44,45}$

One application of the Analyzer has been the development of a test to distinguish non-advanced breast cancer from tumors that are benign. The rationale for the test is the hypercoagulable state seen in cancer patients (Trousseau's Syndrome), resulting from the generation of TF by leukocytes (monocytes). ${ }^{46}$ (Table 4 ).

\section{Fibrin degradation}

\section{Products and fibrin monomer}

DIC can be seen as a two-step process. First, fibrinogen, which is always present in the blood, is activated by any of several mechanisms. This activation leads to an automatic polymerization (chain formation) resulting in fibrin monomer. This is not apparent in a microscope unless the blood is allowed to clot, as in the MOST. ${ }^{47,48}$ The second step is the precipitation or deposition of fibrin (hard clot) by several other mechanisms. One of these is the formation of cross links through the action of factor XIII. Another such mechanism may be poor circulation in an organ already blocked by deposited fibrin. The deposition of precipitated fibrin may be detected microscopically in tissue sections and diagnosed as DIC. ${ }^{49}$

Because fibrin monomer is not readily detected, a chemical test for it is of immense value in diagnosing DIC. Research has indicated that its detection may be very useful in the early diagnosis of DIC and $\mathrm{MAT}^{50}$ There are three fundamental physiologic areas related to blood clotting:

\section{a. The prevention of blood clotting, \\ b. The clotting of blood, \\ c. The removal of clotted blood once it has formed.}

Enzymes are present that are capable of removing (lysing) clotted blood, one of which is plasmin. Another enzyme, plasminogen, is always present in the blood, but is inactive as a proteolytic agent. Plasminogen activator converts plasminogen to plasmin, which can degrade deposited fibrin. This process is not specific for fibrin, however, and other proteins may be affected. When fibrin is degraded (fibrinolysis), fibrin monomer, as well as several other products, are formed. Commercial kits are available for the analysis of fibrin degradation. This test is an indirect measure of the presence of DIC and MAT. $^{51}$

\section{Other tests include}

Protamine Sulfate: Protamine sulfate is a heparin binder sometimes used in surgery for excessive bleeding. The test, which indicates fibrin strands and fibrin degradation products, is conducted in a test tube, with fibrin monomer and fibrin forming early and polymerization of fibrin degradation products occurring later. ${ }^{52}$

Ethanol Gelation: A white precipitate is formed by the addition of ethanol to a solution in a test tube containing fibrin monomer as a degradation product of fibrin, indicating DIC and MAT. ${ }^{53}$

The Mycotoxic/Oxidative Stress Test (MOST): Up to now, blood chemistries have been the primary mode of diagnosis or analysis for 
the presence of pathology. In the view presented here, the bright-field microscope is used to easily and inexpensively reveal a disease state as reflected by changes in certain aspects of blood composition and clotting ability. DIC is characterized by the abnormal presence in the blood of fibrin monomer. When allowed to clot, blood containing such an abnormal artifact will exhibit distortions of normal patterns. The presence in the blood of soluble fragments of the extracellular matrix and soluble fibronectin, as well as other factors, will also create abnormal blood clotting patterns as described below (Monograph 3).

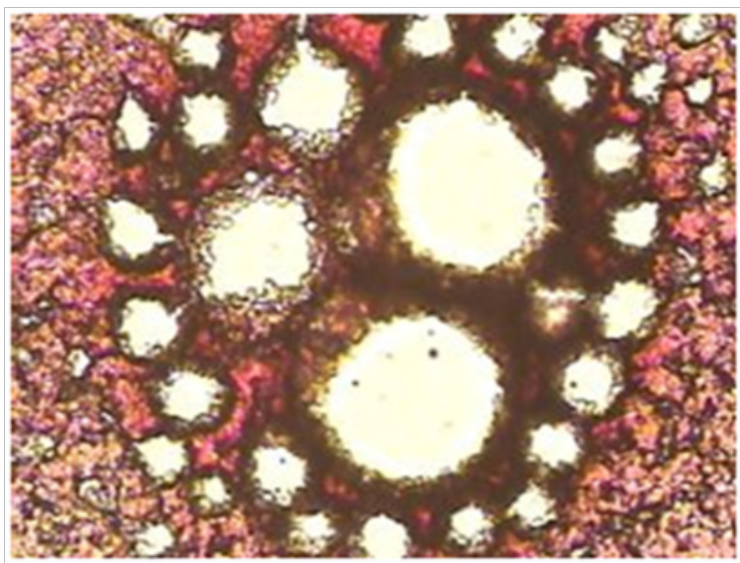

Monograph 3 An abnormal clot with striking 'clear' or white areas or protein polymerization as seen in the hyper coagulated blood of a patient with lower bowel imbalances.

A small amount of blood from a fingertip is contacted with a microscope slide. A series of drops is allowed to dry and clot in a normal manner. Under the compound microscope, the pattern seen in healthy subjects is essentially the same-a dense mat of red areas interconnected by dark, irregular lines, completely filling the area of the drop. The blood of people under mycotoxic/oxidative stress exhibits a variety of characteristic patterns which deviate from normal, but with one striking, common abnormality: "clear" or white areas, in which the fibrin net/red blood cell conglomerate is missing.

Why the fibrin net is missing may be understood from the following: Two peptides-A and B-in the central protein bead of the fibrinogen structure become bound in the cross-linking process. There are two ways this can be configured:

a. Thrombin is capable of activating peptides A and B, resulting in the formation of a polymer loosely held together only by hydrogen bonds;

b. With peptides A and B activated normally, the resulting hard clot is insoluble, indicating that the peptides are linked by covalent bonds. The difference in bonds results from factor XIII, an enzyme which links the two fibrin strands with a glutaminelysine peptide bond.

Additional research has shown that the release of sialic acid from fibrinogen inhibits the action of factor XIII, resulting in a soft, white clot. In addition, acetic aldehyde has been shown to inactivate factor XIII directly. The soft clotting, compounded by other polymeric aggregations (described below), results in clear areas in the dry specimens. In the opposite extreme, high serum levels of calcium, for the purpose of neutralizing MAT, activates factor XIII, leading to excessive cross-linking of fibrin to form a clot harder than normal. This is reflected in the MOST pattern characteristic of definite hypercalcemia- that of a series of cracks in the clot radiating outward from the center, resembling the spokes of a wheel. High serum calcium is the body's attempt to compensate for the acidity of mycotoxic stress by pulling this alkalizing mineral from bone into the blood. This demand creates endocrine stress in turn, because reabsorption of bone is mediated by parathormone (PTH). Therefore, this clotting pattern indicates calcium deficiency and thyroid/parathyroid imbalance (Monograph 4).

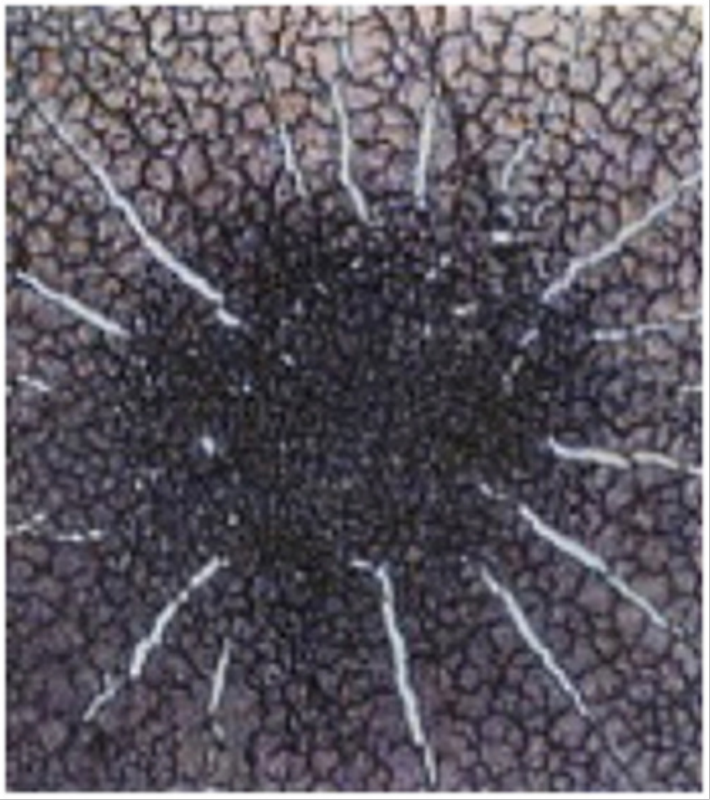

Monograph $4 \mathrm{~A}$ mineral deficiency or more specifically a calcium deficiency pattern associated with an imbalance of the thyroid and/or parathyroid.

Advanced research has shown that there are seven carbohydrate chains in fibrinogen (each terminated by sialic acid). A second action of factor XIII is to ferment a large amount of carbohydrate during clotting. Because carbohydrate is most often water soluble, the loss of this material undoubtedly adds to the insolubility of a clot, while pathological retention contributes to the softness of the abnormal clot.

Clinical experience demonstrates that the MOST is a reliable indicator of exotoxic and mycotoxic stress and, concurrently, of various disorganizing symptomatologies associated with fermentative and oxidative processes. As various cellular degradation occurs, the blood-borne phenomena which accompany such symptoms as diabetes, arthritis, heart attack, stroke, atherosclerosis and cancer show up in the MOST, often with sialic acid beads in the clear areas of polymerized proteins. (Determination of the liberation of sialic acid from carbohydrate has been approved by the U.S. Food and Drug Administration as an accepted indicator for cancer, and is clinically available) (Monograph 5).

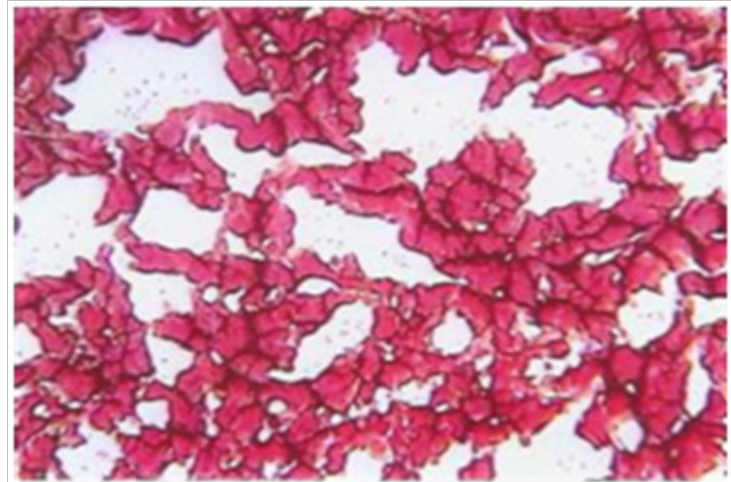

Monograph 5 Sialic acid beads are seen inside the protein polymerization of the hypocoagulated blood as black. 
The extent and shape of the clear areas are reflective of particular symptomatologies which have arisen from the way in which the disease condition manifests in a given individual. This observation is borne out by having the patient undergo appropriate therapy. With success of treatment based on the patient's freedom from symptoms, sense of well-being, and live blood exams discussed in the main text of Sick and Tired, Reclaim Your Inner Terrain, Appendix C, ${ }^{9}$ repeated analysis with the MOST reveals a progressively improving clotting pattern (Monograph 6A \& 6B).
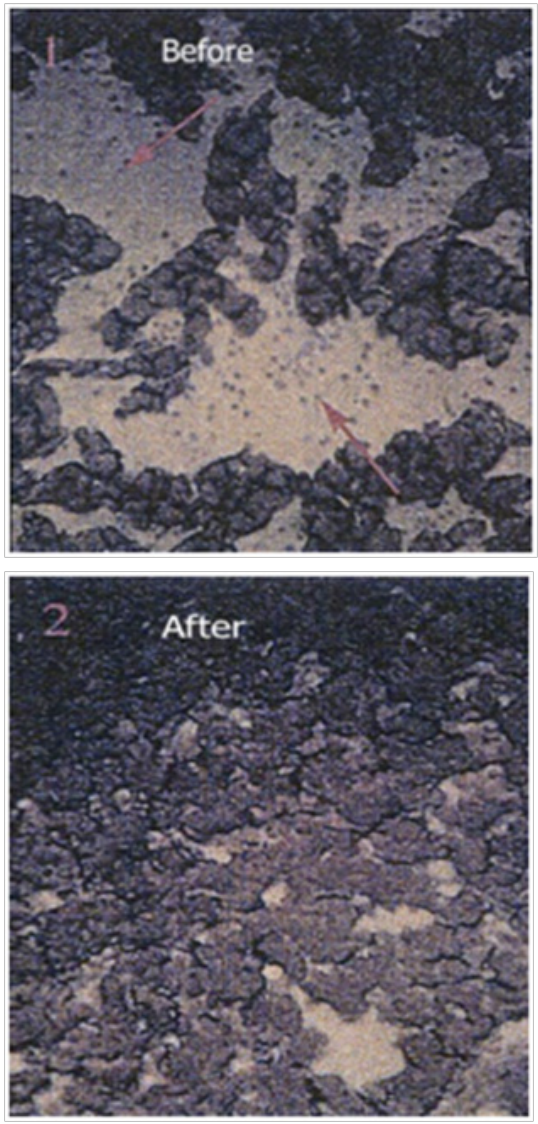

Monograph 6A \& 6B Medically diagnosed cancer patient with large polymerized protein pools (PPP) in the hypo-coagulated blood above. In the picture below PPP's have significantly reduced in size and the blood is moving to a more hyper-coagulated state as a result of reducing acid loads with an alkaline lifestyle and diet. ${ }^{9,53}$

Because of its very nature, the MOST is eminently suited to reveal and measure the presence in the blood of abnormal substances, clotting factors, and disorganization of cells due to an inverted way of living, eating, and thinking, which gives rise to MAT. The MOST indicates both the direct and indirect activity of MAT on blood clotting, endothelium, and the extracellular matrix (described next), as well as on biochemical pathways, including hormonal ones. The generation of excessive MOBS in response to EMPO and MAT, the inability that accompanies all degenerative symptoms to neutralize or eradicate EMPO and MAT, and the recognized hyper- and hypocoagulable states seen in various symptomatologies, will beyond doubt be revealed in the MOST (Monograph 7A \& 7B).

As mentioned, hormones are easily fermented, and this will show up as a hypocoagulated blood pattern in the MOST. It is my opinion, this hypocoagulated blood appears in the MOST as misty clouds of protein polymerizations throughout the clot, as seen in the accompanying picture (Monograph 8).
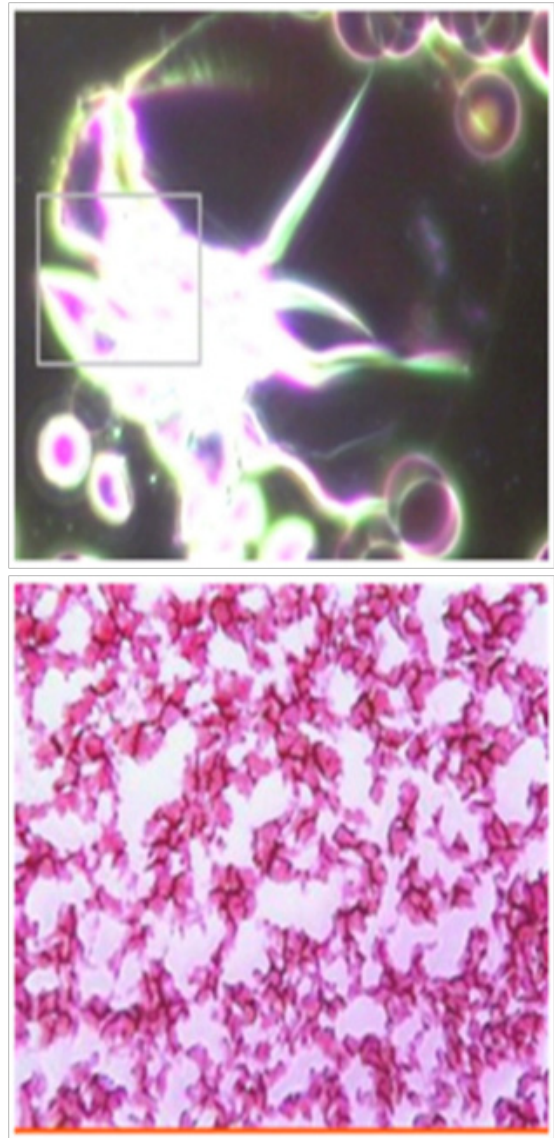

Monograph 7A \& 7B Medically Diagnosed HIVIAIDS micrograph showing above an Aspergullus niger mould crystal using dark field microscopy and below a hypocoagulated blood clot with systemic protein polymerizations measuring in excess of 40 microns using bright field microscopy.

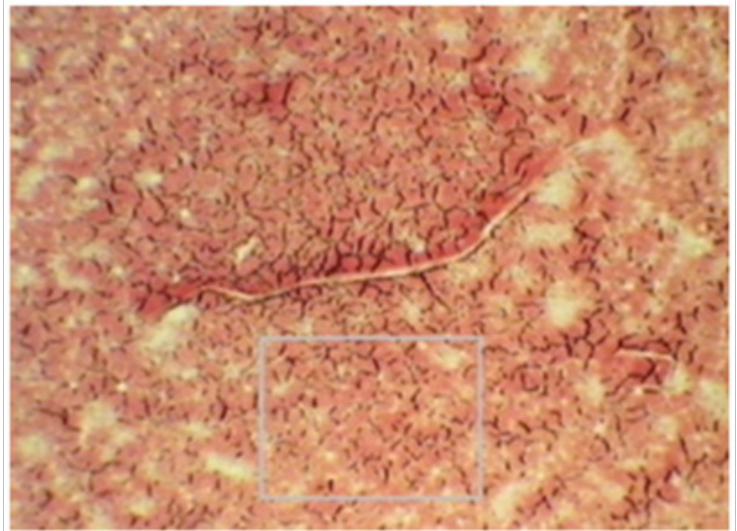

Monograph 8 Poor fibrin interconnection in the clot associated with endocrine or hormonal imbalance.

The MOST from solubilized extracellular matrix: There is now a clearer picture of the biochemical rationale for correlating abnormal blood clotting patterns with the presence of degenerative symptoms. A link between symptoms and the distorted clotted blood patterns has been delineated in the MOST.

Another reason for the abnormal clotting patterns accompanying pathological states, in addition to insufficient bonding of fibrinogen peptides as seen in the MOST, is presence in the blood of watersoluble fragments of the extracellular matrix. 
Extracellular matrix degradation by MAT: The extracellular matrix (EM) is a three-dimensional gel, binding cells together and composed of five or more major constituents: collagen (protein), hyaluronic acid (polysaccharide), proteoglycans (pro-tein/ polysaccharide), fibronectin and laminin. Also included are glycosaminoglycans and elastin. ${ }^{54}$ In every degenerative disease studied by this author, evidence has been found for MAT activity destructive of EM.

One of the proteolytic enzymes activated in response to EMPO and MAT is alpha-1 antitrypsin (capable of neutralizing MAT), normally not active in the presence of the enzyme trypsin. The active portion of this anti-exotoxin and antimycotoxin contains the amino acid methionine, which includes a C-S-C linkage. When chelated by the hydroxyl radical (one of the MOBS oxidants), methionine's central sulfur atom acquires one or two oxygen atoms (forming the sulfone or sulfoxide respectively). The fermentation of methionine is a secondary effect of immune response to an alarming situation, intended to neutralize MAT and prevent degradation of the EM. Once alpha-1 antitrypsin is exhausted, MAT will have more access to the EM. If the EM is damaged beyond repair, then the enzyme trypsin is released to disorganize and recycle the cells involved. ${ }^{54}$

A similar scenario holds for the enzymes collagenase and elastase. Thus, the absence of alpha- 1 antitrypsin in the presence of EMPO and MAT activates three enzymes which degrade the extracellular matrix. Degradation of the EM by enzymes and MAT puts into the blood the water-soluble fragments (proteins and glycoproteins) of normally insoluble EM components (Table 12). The presence of these fragments modifies the normal clotting pattern (described below), as seen in the M/OST, and is therefore an indication of EM degradation, which is always found with degenerative symptoms. (Also present is fibrin monomer, which has been found in the blood of patients suffering from collagen disease ${ }^{55}$ (Table 12).

Fibronectin is a molecule in EM having several binding sites for various long-chain molecules- heparin (a sulfonated polysaccharide) and collagen, for example. As such, it functions as a cellular glue, binding cells together as well as various components of the EM. A soluble form of fibronectin is normally found free in the blood, and enters into the formation of a blood clot through the action of factor XIII. This form of fibronectin binds to fibrin. Elevated, bound-serum fibronectin results from EM fragmentation by MAT, and accompanies degenerative symptoms such as arthritis and emphysema (collagen diseases).

Water-soluble fragments of the EM bound by fibronectin form a three-dimensional network or gel in the pathologically clotted blood (fibrin and components of the blood clotting cascade). Since fibronectin binds to both fibrin and collagen, the two polymeric networks are superimposed and intermingled, resulting in a modification of the normal clotting pattern. Exactly how the pattern is modified depends upon the nature of the collagen abnormally present, the nature and extent of hyaluronate present, and the degree to which EM fibronectin has been released by MAT.

\section{Conclusion}

Thus, it is easily seen that there are many forms which the pattern of clotted blood may take, depending on the individual and the internal terrain that produced the modifying substances. The MOST reveals not only the presence of exotoxic and mycotoxic stress, but indicates as well the nature of the symptom(s) resulting from the stress (Table 13). Since MAT underlie the entire complex of events which degrade the extracellular matrix, I must conclude that the absence of these exotoxins, endotoxins and mycotoxins would provide substantial improvements in tissue integrity and the overall physiology and functionality of the organism or animal and human.

\section{Acknowledgments}

None.

\section{Conflicts of interest}

Author declares there are no conflicts of interest.

\section{Funding}

None.

\section{References}

1. Jones TW. Observations on some points in the anatomy, physiology and pathology of the blood. British Foreign Medical Review. 1842. p.1-24.

2. Trousseau A.Phlegmasis alba delens. Clinque Medicale de L'Hotel Dieu de Paris. 1865;3:94.

3. Virchow R. Hypercoagulability: A review of its development, clinical application, and recent progress. Gesammelte Abhandlungen our Wussenschaftichen Medizin. 1856;26:477.

4. Rapaport SI. Blood Coagulation and its Alterations in Hemorrhagic, and Thrombotic Disorders. West J Med. 1993;158(2):153-161.

5. Be Champ A. The Blood and Its Third Anatomical Element. Hikari Omni Publishing, USA. 1999.

6. Schwerdtle C, Arnoul F, Enerlein G. Introduction to Darkfield Diagnostics. Semmelweis-Verlag, Germany. 2006.

7. The Harper Collins Illustrated Medical Dictionary. 1994. p.13.

8. Hamilton PJ, Stalker Al, Douglas AS. Disseminated Intravascular Coagulation: A Review. Journal of Clinical Pathology. 1978;31:609-619.

9. Young RO. Sick and Tired, Reclaim Your Inner Terraine. Woodland Publishing, USA. 1999.

10. Hawk BO, Thoma GE, Inkley JJ. An Evaluation of the Bolen Test as a Screening Test for Malignancy. Cancer Res . 1951;11(3):157-160.

11. Uchida K. Role of Reactive Aldehyde in Cardiovascular Diseases. Free Radic Biol Med. 2000;28(2):1685-1696.

12. Chang JC, van der Hoeven LH, Haddox CH. Glutathione reductase in the red blood cells. Ann Clin Lab Sci . 1978;8(1):23-29.

13. Kutzing MK, Firestein BL. Altered Uric Acid Levels and Disease States J Pharmacol Exp Ther. 2008;324(1):1-7.

14. Claudino M, Ceolin DS, Alberti S, et al. Alloxan-Induced Diabetes Triggers the Development of Periodontal Disease in Rats. Plos One. 2007;2(12):e1320

15. Young RO. Alkalizing Nutritional Therapy in the Prevention and Reversal of any Cancerous Condition. IJCAM. 2015;2(1):00046.

16. Fernandes HP, Cesar CL, Barjas Castro Mde L. Electrical properties of the red blood cell membrane and immunohematological investigation. Rev Bras Hematol Hemoter. 2011;33(4):297-301.

17. Harris JO. A study of The Relationship Between the Surface Charge and the Absorption of Acid Dyes by Bacterial Cells. J Bacteriol. 61(6):649-652.

18. Young RO. Metabolic and Dietary Acids are the Fuel That Lights the Fuse that Ignites Inflammation that Leads to Cancer. Int J Complement Alt Me. 2016;3(6):00094.

19. Snaders R. Did Bacteria Spark Evolution of Multicellular Life? Berkeley News, USA. 2012 
20. Wenner M. Humans Carry More Bacterial Cells than Human Ones Scientific American, USA. 2007.

21. Animals and humans respond to MAT as a poison.

22. Morrison DC, Ulevitch RJ. The effects of bacterial endotoxins on host mediation systems. Am J Pathol. 1978;93(2):526-618.

23. Van Deventer SJ, ten Cate JW, Tytgat GN. Intestinal endotoxemia Clinical significance.Gastroenterology. 1988;94(3): 825-831.

24. Morrison DC, et al. op. cit.

25. Hu T, Bach RR, Horton R, et al. Synthesis of tissue factor messenger RNA and procoagulant activity in breast cancer cells in response to serum stimulation. Thromb Res. 1993;72(2):155-168.

26. Mackman N, Brand K, Edgington TS. Lipopolysaccharides-mediated transcriptional activation of the human tissue factor gene in THPmonocytic cells requires both activator protein 1 and nuclear factor kappa B binding sites. J Exp Med. 1991;174(6):1517-1526.

27. Yamada O, Charles F Moldow, Sacks T, et al. Deleterious effects of endotoxins on cultured endothelial cells: An in vitro model of vascular injury. Inflammation. 1981;5(2):115-126.

28. M Colucci, G Balconi, R Lorenzet, A Pietra, et al. Cultured human endothelial cells generate tissue factor in response to endotoxin. J Clin Invest . 1981;71(6):1893-1896.

29. Cho TH et al. Effects of Escherichia coli toxin on structure and permeability of myocardial capillaries. Acta Pathol Jpn. 41(1):12-18.

30. Margolis J. The interrelationship of coagulation of plasma and release of peptides. Annals of the New York Academy of Sciences. $1963 ; 104: 133-145$.

31. Morrison DC, et al. op. cit.

32. Alberts B, et al. Molecular Biology of the Cell. (2nd edn)Garland Publishing, New York, USA. 1986. p.818.

33. Nachman RL, et al. Hypercoagulable states. Annab of Internal Medicine. $1993 ; 119: 819$

34. Tallman MS, Hakimian D, Kwaan HC, et al. New insights into the pathogenesis of coagulation dysfunction in acute promyelocytic leukemia. Leuk Lymphoma. 1993;11(1-2):27-36.

35. Silberberg JM, Gordon S, Zucker S. Identification of tissue factor in two human pancreatic cancer cell lines. Cancer Res. 1989;49(19):5443-5447.

36. Grimstad IA, Prydz H. Thromboplastin release, but not content, correlates with spontaneous metastasis of cancer cells. Int $J$ Cancer. 1988;41(3):427-431.

37. Gunji Y, Gorelik E. Role of fibrin coagulation in protection of murine tumor cells from destruction by cytotoxic cells. Cancer Res . 1988;48(18):5216-5221.

38. Sugiyama S, Hayakawa M, Hanaki Y, et al. The role of leukotoxin (9, 10- epoxy-12-octadecenoate) in the genesis of coagulation abnormalities. Life Sci. 43(3):221-227.
39. White A, et al. Principles of Biochemistry. McGraw-Hill Book Co., New York, USA. 1964. p.648

40. Mueller HE, et al. Increase of microbial neuraminidase activity by the hydrogen peroxide concentration. Experientia. 1972;23:397.

41. Robert OY. Fermentology and oxidology. The study of fungus-produced mycotoxic species and the activation of the immune system and release of microzymian oxidative buffering species (MOBS). Inner Light Biological Research Foundation, Alpine, Utah, USA. 1994.

42. Chandler WL, Schmer G. Evaluation of a new dynamic viscometer for measuring the viscosity of whole blood and plasma. Clin Chem. 1986;32(3):505-507.

43. Saleem A, Blifeld C, Saleh SA, et al. Viscoelastic measurement of clot formation: A new test of platelet function. Ann Clin $\mathrm{Lab} \mathrm{Sci}$. 1983;13(2):115-124.

44. Spillert CR, Passannante MR, Salzer Pagan JE, et al. Altered coagulability: An aid to selective breast biopsy. J Natl Med Assoc. $1983 ; 85(4): 273-277$.

45. Bowie EJ, Owen CA. The clinical pathology of intravascular coagulation. Bibl Haematol. 1983;49:217-224.

46. Berghaus MG, Eckhardt T. The role of granulocytes in the activation of intravascular coagulation and the precipitation of soluble fibrin by endotoxin. Blood. 1975;45(5):631-641.

47. Bick RL. Disseminated intravascular coagulation. Hematology/ Oncology Clinics of North America. 1993;6:1259.

48. Bredbacka S, Blombäck M, Wiman B, et al. Laboratory methods for detecting disseminated intravascular coagulation (DIC): New aspects. Acta Anaesthesiol Scand. 1993;37(2):125-130.

49. Sigma Diagnostics, St. Louis, USA.

50. Gurewich V, Hutchinson E. Detection of intravascular coagulation by a serial-dilution protamine sulfate test. Ann Intern Med. 1971;75(6):895-902.

51. Breen FA, Tullis JL. Ethanol gelation: A rapid screening test for intravascular coagulation. Ann Intern Med. 1970;69(6):1197-1206.

52. Hay ED. Cell Biology of Extracellular Matrix. Plenum Press, Springer US, New York. 1991. p.653.

53. Carp H, Janoff A. In vitro suppression of serum elastase- inhibitory capacity by ROTS generated by phagocytos- ing polymorphonuclear leukocytes. J Clin Invest. 1979;63(4):793-797.

54. Wilson CL, Schwarzbauer JE. The alternatively spliced V region contributes to the differential incorporation of plasma and cellular fibronectins into fibrin clots. J Cell Biol. 1992;119(4):923-933.

55. Fernandes HP, Cesar CL, Barjas Castro Mde L. Electrical properties of the red blood cell membrane and immunohematological investigation. Rev Bras Hematol Hemoter. 2011;33(4):297-301 